\title{
Welcher Biomarker sichert besser die Diagnose?
}

Fragestellung: In dieser Arbeit sollte geprüft werden, ob der Biomarker GDF-15 bezüglich Sensitivität und Spezifität für eine Mitochondrienerkrankung des Erwachsenen besser geeignet ist als FGF-21.

Hintergrund: Mitochondriale Erkrankungen können auf vielfältige Weise diagnostiziert werden. Zum einen kann über Laktatbestimmungen im Serum oder Liquor durch die Analyse von Kreatinkinase oder der Laktat/Pyruvat-Ratio im Serum ein Hinweis auf eine mögliche mitochondriale Störung gewonnen werden. Jüngere Arbeiten zeigen, dass der Fibroblastenwachstumsfaktor 21 (FGF-21) im Mausmodell gut mit einer mitochondrialen Myopathie korreliert. Später konnte nachgewiesen werden, dass dies auch für Patienten mit mitochondrialer Myopathie galt. Jüngere Arbeiten zeigten dann, dass auch der "growth differentiation factor-15“ (GDF-15) ein potenzieller Indikator für das Vorliegen einer mitochondrialen Erkrankung ist. Insbesondere bei Patienten mit der Punktmutation 3243 des mitochondrialen Genoms, das heißt MELAS-Patienten, konnte eine positive Korrelation gefunden werden.

Patienten und Methodik: Insgesamt wurden 66 erwachsene Patienten untersucht, von denen 20 eine nicht mitochondrial bedingte neuromuskuläre Erkrankung hatten und als Kontrolle dienten, und 54 Patienten mit

Davis RL, Liang Ch, Sue CM. A comparison of current serum biomarkers as diagnostic indicators of mitochondrial diseases. Neurology 2016; 86: 2010 -5 einer mitochondrialen Erkrankung. Die Patienten hatten entweder eine genetische Diagnostik erfahren, die für eine mitochondriale Erkrankung spricht oder in der
Muskelbiopsie fanden sich typische mitochondriale myopathische Veränderungen. Die Patienten waren zwischen 20 und 84 Jahre alt, etwa 58 \% waren Frauen. Von den 54 Patienten mit der mitochondrialen Erkrankung wiesen 27 typische Genveränderungen und 27 typische Muskelveränderungen auf. Neun der genetisch determinierten Erkrankten zeigten auch eine typische Muskelpathologie. Bei allen Patienten war im Vorfeld FGF-21 bestimmt worden. Jetzt erfolgte zusätzlich eine Bestimmung der GDF-15-Serumspiegel im Serum.

Ergebnisse: Die Kontrollgruppe hatte den niedrigsten, die Krankheitskontrollgruppe einen mittleren Wert und die Patienten mit einer Mitochondrienerkrankung die höchste Konzentration von GDF-15. Im Schnitt lagen die GDF-Konzentrationen hier 2- bis 4,6-fach höher als bei den Kontrollen. Auch bei den Analysen von FGF-21 zeigte sich eine signifikante Differenz zwischen den drei Untersuchungsgruppen. Die Sensitivität war für beide Marker hoch. Für die Patienten, die genetische Mutationen aufwiesen, war die Sensitivität für GDF-15 höher als für FGF-21. Insgesamt war der prädiktorische Wert für GDF-15 deutlich höher als für FGF-21. GDF-15 war ein besserer Prädiktor mit einer Sensitivität von 77,8 \% gegenüber 68,5\% für FGF21. Eine Analyse beider Faktoren erhöhte die prädiktorischen Werte für eine mitochondriale Erkrankung nicht.

Schlussfolgerungen: Bei FGF-21-positiven Patienten stellte GDF-15 einen sehr wertvollen Parameter dar. GDF-15 war ein besserer Indikator für vererbte mitochondriale Erkrankungen unabhängig vom Phänotyp, während FGF-21 sich bei mitochondrialen Erkrankungen mit Muskelmanifestation als der bessere Biomarker erwies.

\section{- Kommentar von Heinz Reichmann, Dresden}

\section{Eine neue Möglichkeit der diagnostischen Absicherung eröffnet}

Das vorgestellte Kollektiv ist dahingehend besonders, dass lediglich Erwachsene untersucht wurden. Es wurde eine Kombination von Patienten, die als mitochondrial erkrankt galten, weil sie zum einen eine genetische Abnormität oder alternativ eine typische Muskelpathologie aufwiesen, in diese Studie mit aufgenommen. Die Autoren gehen somit mit Recht davon aus, dass hier Fehldiagnosen nahezu unmöglich sind. Interessant ist, dass japanische Autoren in ihrer Arbeit nachwiesen, dass dort GDF-15 der bessere Indikator für eine Muskelmanifestation bei Mitochondriopathie darstellt, sodass hier unterschiedliche Ergebnisse gewonnen wurden.

Hervorzuheben ist, dass diese Studie erneut belegt, dass neben den oben genannten, zum Teil invasiven Diagnosemethoden für Patienten mit mitochondrialen Zytopathien sich durch die Etablierung der beiden Biomarker FGF-21 und eventuell insbesondere durch die Etablierung des GDF-15 eine neue Möglichkeit zu diagnostischen Absicherung bei Verdachts- diagnose auf eine mitochondriale Zytopathie eröffnet. Sobald diese Methoden frei zur Verfügung stehen und weitere Arbeitsgruppen Erfahrungen damit gesammelt haben, werden wir die Wertigkeit solcher Analysen im Vergleich zur genetischen Analyse oder der muskelhistologischen Aufarbeitung noch besser abschätzen können.

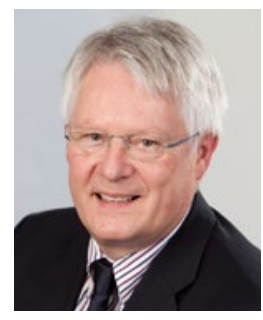

Prof. Dr. med. Heinz Reichmann, Dresden

Direktor der Klinik und Poliklinik für Neurologie, Klinikum Carl Gustav Carus der TU Dresden, Dekan der Medizinischen Fakultät. E-Mail: heinz.reichmann@ uniklinikum-dresden.de 